\title{
Oil Energy Exploration
}

\section{Application of Faults Identifying Technique In Oilfields}

\author{
Dayou $\mathrm{Xu}^{1}$ \\ College of Earth Science, Northeast Petroleum University \\ Daqing, China \\ Leonidas_813@hotmail.com
}

\author{
Wei Wei ${ }^{2}$ \\ College of Earth Science, Northeast Petroleum University \\ Daqing, China \\ Hunqiu8854@hotmail.com
}

\begin{abstract}
Faults play an important role in the complex faultblock reservoir. Fine fault interpretation and combination matters a lot. Aiming at the difficulty of the complex fracture system and fault interpretation, this paper bases on the union of wells and seismic, make full use of spectral decomposition, seismic curvature, coherence analysis and mixing frequency with 3 colors, then governs the whole interpretation with 3D structural model. With the use of various methods, we start to identify faults. This technique achieves a good effect in research area, especially for the small fault identifying, overcomes the lack of resolution in seismic data, increases the interpretation accuracy, finds and delineates a batch of small faults, guides the plane distribution and space intersection relation of faults, achieve the goal of fine structural interpretation.
\end{abstract}

Index Terms-Faults identifying, mixing frequency with 3 colors, coherence analysis, spectral decomposition, union of wells and seismic.

\section{INTRODUCTION}

As we know, petroleum resource becomes the most vital energy resource in the world today and petroleum detection plays an important role in petroleum exploitation. With the development of technology and the growing demand for the production, many oilfields enter the development stage. Fine structural interpretation is the foundation of field development project design.

Profile fault interpretation is the conventional method, based on the features of composite wave: amplitude mutation, fault surface waves, the increase or decrease of the number of lineup, lineup leap, strong phase transformation, messy reflection and blank tapes. But with the limit of the resolution of seismic data, when the fault displacement is less than or close to $1 / 4$ wavelength, the diastrophism is not obvious in the profile. We can't achieve our goal of fine interpretation only with the profile identifying, therefore we introduce geophysical technology of the union of wells and seismic, seismic attribute, with the guidance of $3 \mathrm{D}$ structural modeling, to develop the research of fault identifying in the studied area.

\section{SURVEY OF RESEARCH AREA}

Beizhong Sub-Depression is located in the south of Beier Sag in Hailaer Basin, with the Bulehongsi Uplift Zone on its east and the Sudeerte Uplift Zone on its west. It is a junior fault depression which is belonged to Beier Concave, with the distribution area of $800 \mathrm{~km} 2$. From top to bottom Beizhong Sub-Depression develops Quaternary, Tertiary Huchashan Group, Cretaceous Qingyuangang Group, Lower Crataceous Yinmin Group, Damoguaihe Group, Nantun Group, Tongbomiao Group and Triassic Budate Group, among them Nantun Group is the main oil layer with 18 wells on production.

\section{FAULTS IDENTIFYING TECHNIQUE}

The template is used to format your paper and style the text. All margins, column widths, line spaces, and text fonts are prescribed; please do not alter them. You may note peculiarities. For example, the head margin in this template measures proportionately more than is customary. This measurement and others are deliberate, using specifications that anticipate your paper as one part of the entire proceedings, and not as an independent document. Please do not revise any of the current designations.

\section{A. Union of Wells and Seismic Data}

Fault identifying in the seismic profile is the main method of fault interpretation based on the features of the composite waves. Seismic data gives the information in both horizontal and vertical direction, while its resolution is very low. Research area has entered the development stage with dense wells. The interpretation of breakpoints in wells is affected by well spacing density. The combination of well breakpoints is multiplicity, and there exists isolated breakpoint. Therefore we introduce the method of the union of wells and seismic. Combining the faults in the profile with the breakpoints in wells, we start the interactive interpretation to ensure the accuracy and rationality of the fault interpretation and rationality. Fig 1 is the comparison diagram of seismic interpretation and seismic-well interpretation. From the picture we can see that 2 faults are found and 4 breakpoints are seated. With the guidance of the breakpoints in wells, we start the interpretation in the profile. In this way, we improve the accuracy. It is applicable in the whole research area. 

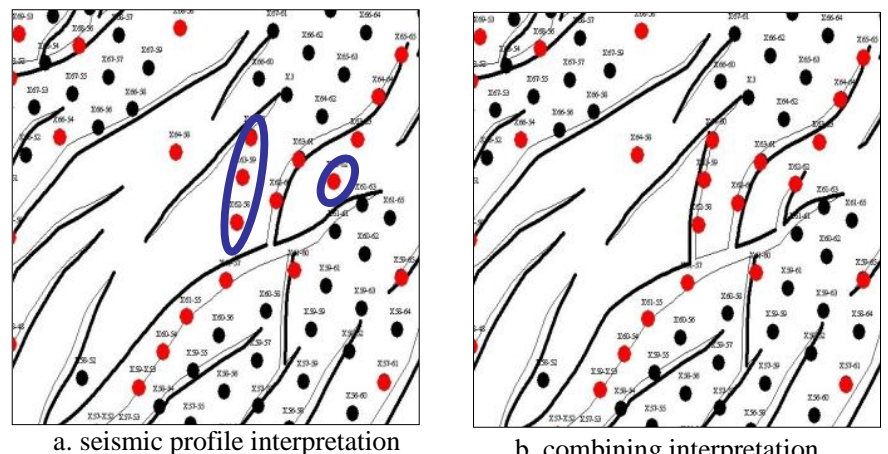

Fig. 1.the union of wells and seismic data

\section{B. Spectral Decomposition}

Spectral decomposition is a common method used in faults identifying[1]. Its principle is to turn the time domain seismic data into the frequency domain to get the amplitude spectrum and phase spectrum. Based on this, we can acquire tuning data. If $g(t)$ is the time domain seismic data and $G(f)$ is the corresponding frequency domain data, then there is:

$$
G(f)=\int_{<\infty}^{\infty} g(t) e^{2 \pi f t} d t
$$

This transformation belongs to DFT, and it calculates every amplitude value from the high-frequency to low-frequency. Its mathematical expression is:

$$
A(k)=\sum_{i=0}^{N<1} a(j)\left(\cos \frac{2 \pi j k}{N}+i \sin \frac{2 \pi j k}{N}\right)
$$

And, $i=\sqrt{-1} ; a(j)$ is the Amplitude value when the time is $j ; A(k)$ is the Amplitude value when the frequency is; $N$ is sampling number in the time window.

Seismic data in different frequency bands has different sensitivity to the faults[2]. After the spectral decomposition, we find some single frequency has sensitivity to the faults in research area. Fig 2 shows $20 \mathrm{~Hz}, 35 \mathrm{~Hz}$ and $50 \mathrm{~Hz}$ frequency slices. The master frequency of seismic data is $35 \mathrm{~Hz}$.From the picture we can tell, when it is master frequency, there is a batter display of the faults. This method can provide us a true reflection of the fracture and guide the flat combination of faults.

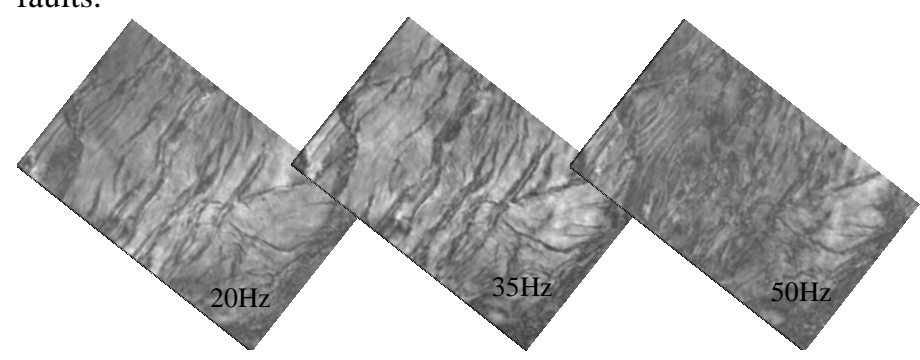

Fig. 3.Frequency slices

\section{Curvature Analysis}

Curvature attribute is a geometric property of seismic attributes. In mathematics, curvature refers to any of a number of loosely related concepts in different areas of geometry. In geophysics, curvature attribute is used to calculate the distribution of geological body in the geometric space. With this, we can identify faults and fractures[3]. Curvature attribute is divided into 2D and 3D.2D curvature is based on T0 map without the guidance of seismic data, compared with 3D curvature which is based on seismic data, the analysis result can not reflect the real structure underground. In this way we turn seismic data into dip data, then calculate the curvature of random points, at last, get the $3 \mathrm{D}$ curvature data. Based on $3 \mathrm{D}$ curvature this paper calculates the most-positive curvature and the negative curvature minimum. From Fig 3, we find the analysis result that the most-positive curvature is better then the negative curvature minimum. This is an effective method for faults identifying.

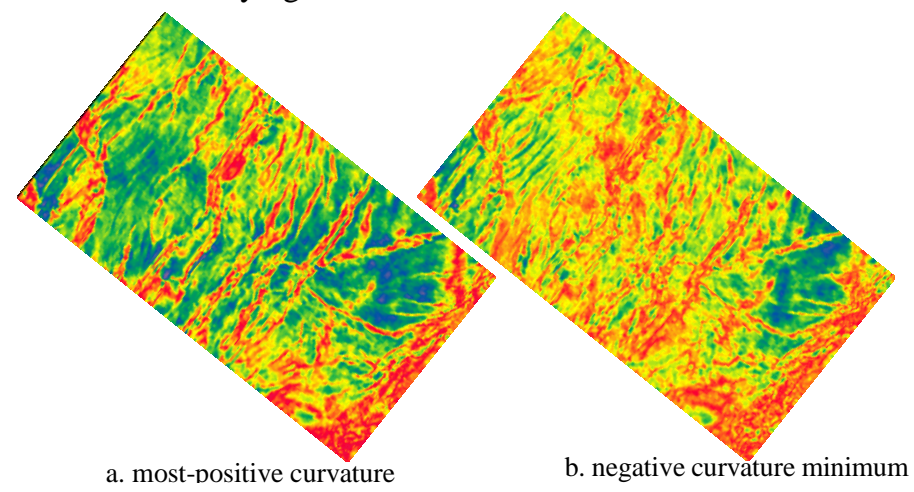

Fig. 2. Curvature analysis results

\section{Coherence Analysis}

Based on calculating the correlation of adjacent seismic traces, mainly the similarity of seismic waveform[4], coherence analysis technique looks for abnormal phenomena, analyses the coherence, accordingly identify the structural features. Faults have a strong influence on the spreading seismic wave in amplitude, phase and frequency. Compared with the adjacent seismic waves, they are dissimilarity. According to this, we calculate the correlation of seismic waves in both horizontal and vertical direction, and then get the coherence data. The coherency cube is sensitive to faults. Based on the fine interpretation of layers, this paper extracts the coherence attribute along the layer. In this way we can increase the resolution and find the small fault easily. As Fig 4 shows that the coherence analysis contributes to Faults identifying, especially the small faults.

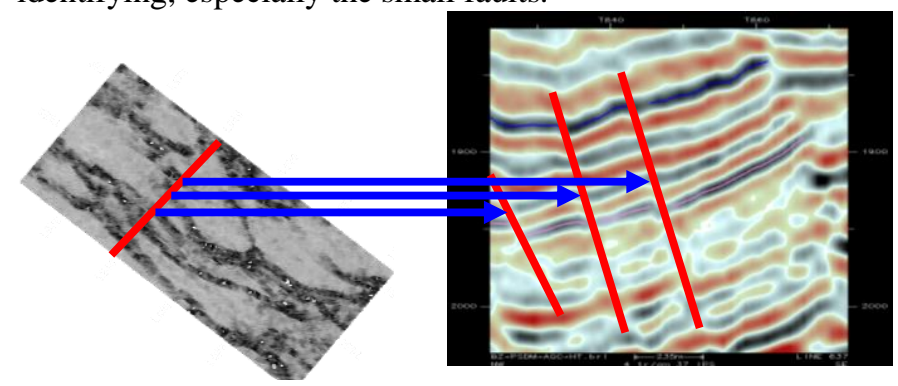

Fig. 4.Coherence Analysis 


\section{E. Frequency Mixing With 3 Colors}

Frequency mixing with 3 colors combined with 3D seismic pixel processing technology and seismic attribute is used to identify lithology and fault in petroleum exploration. With the fusion of seismic attributes, we can tell the structural attitude and the distribution of the lithology.

The resolution of seismic data limited by its signal bandwidth is low, and it's hard to identify small faults. Therefore, we interpreters use kinds of seismic attributes to increase the identification ability. Single attribute has different sensibility to the fault based on digital signal processing theory. Sometimes they show the blurry information and it's hard to interpret the small faults. Consequently here comes the frequency mixing with 3 colors. This method can improve the ability of seismic attributes to identify the fracture. With the fusion of three seismic attributes in RGB 、 HSV and CMY, we adjust the weights of each attribute to highlight the target for the evaluation of the mixing effect. The mixing attribute has a better result compared with every single one. Fig 5 shows us the structure attribute, RMS attribute, amplitude energy attribute and the mixing attribute. From the picture we can tell that the mixing attribute gives a better response to the fault, increases the response of Faults identifying. It is widely used in the research area, and has a good effect.

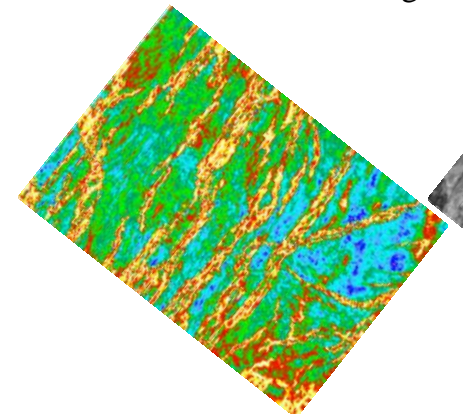

Structural attribute

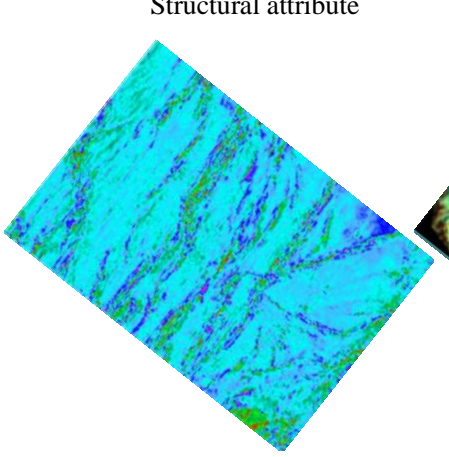

amplitude energy attribute

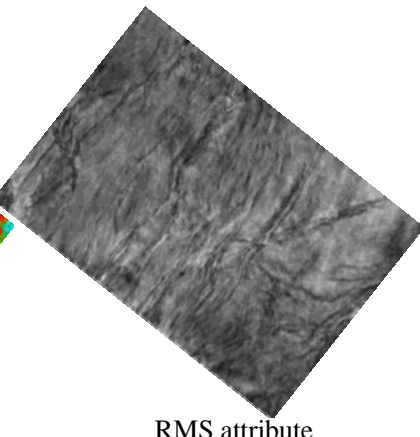

RMS attribute

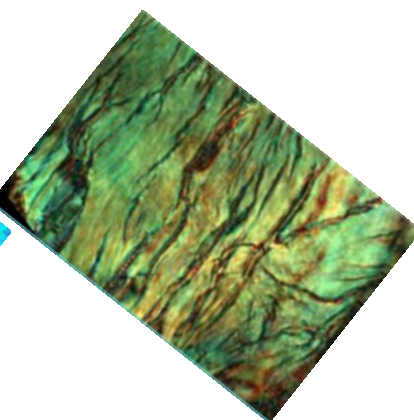

Frequency Mixing With 3
Fig. 5.Frequency mixing with 3 colors comparison

\section{F. 3D Geological Sstructure Modeling Technology}

Structure modeling is one of the 3D geological modeling. According to the information of points and lines, the major theories guide to build the spatial distributions of the layers and fault surfaces, in order to research the feature system and reflect the situation of the geological structure more accurately and visually with the model, based on seismic and drilling data and the applications of the basin structural deformation geometry and kinematics principles[5]. The establishment of the structure modeling mainly sets the fault data, layer surface data and the layering and fault points data of the single well as the input data source, and then the fault modeling and layer surface modeling are built with the application of the 3D seismic data volume to examine and control the fault modeling[6].

On the basis of the seismic data interpretation and $\log$ interpretation, the structure modeling is established with the applications of the union of logging and seismic data, the combination of fault points, the fault modeling and layer simulation[7]. And then the fault points of the wells around the fault modeling, which are attributed to one fault, is observed in 3D space. The fault points are filtered by the simulation initial fault surface figure and the faults are combined deeply in profile under the control of the fault section map, which is perpendicular to the fault. The application effects are analyzed below:

The establishment of the fault space relation: as to the branching faults and intersecting faults, under the circumstance of keeping consistent in the inclinations of the pillar of the branching faults or secondary faults, extend the branching faults or secondary faults to the major faults, the intersection relations and the spatial combinations are confirmed.

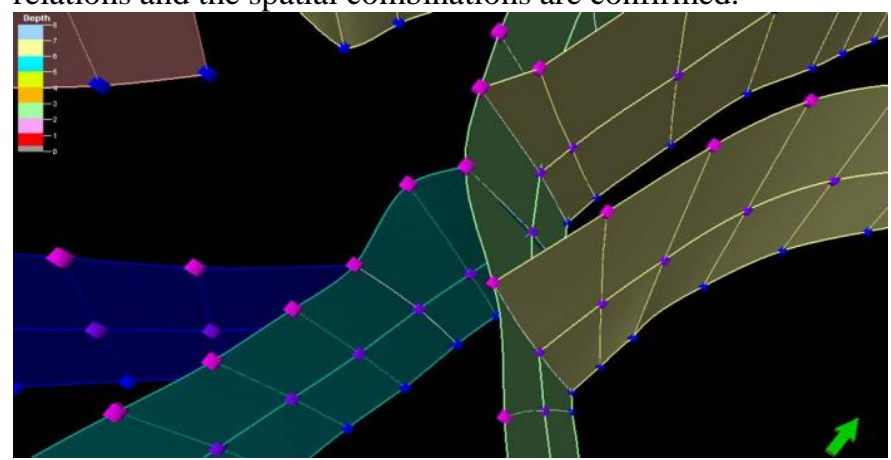

Fig. 6.The establishment of the fault

\section{CONCLUSIONS}

Through the integrated applications of the several identification technologies of faults, it is better to identify the faults in seismic interpretation and it is easier to understand of the fault system, and improve the local structure forms. The synthesis technique had obtained the good application effects. The results are shown below:

1) There are several irrationalities in the conventional profile interpretation, it is difficult to identify the small faults and ascertain the distribution of the complex fault system. It is better to combine other Relevant Information and all-round comprehensive research of the seismic data in the actual seismic interpretation, and then the ability of interpreting faults would be improved.

2) Through the comprehensive applications of Spectral decomposition technique, Coherence analysis technique and seismic curvature attribute technology, the fault characterizes 
were more objectively reflected in the seismic data, the weakness of the lack of Seismic resolution were improved and the effects of the fault identification were better. Meanwhile it has a guiding significance for the flat combination of the faults.

3) With the difference from the traditional RGB color analysis technique ,the new technique of the frequency mixing with 3 colors was introduced into the research, which was combined the HSV and CMY color pattern analysis. This dominant combination improved the ability of the faults dramatically.

4) On the basis of the detailed fault interpretation, with the combination of the log interpretation results, the $3 \mathrm{D}$ geological structure modeling technology was applied to guide the fault identification integrally, which started from the three aspects of point, plane and solid, correct the breakpoints, combine the faults reasonably and construct the intersection relations of the faults in the space. Not only the method obtained a good application effect and improved the rationality and credibility of the fault, but also had the great significance in the development.

\section{COPYRIGHT FORMS}

This is an original paper written by Dayou $\mathrm{Xu}$,edited by Wei Wei.
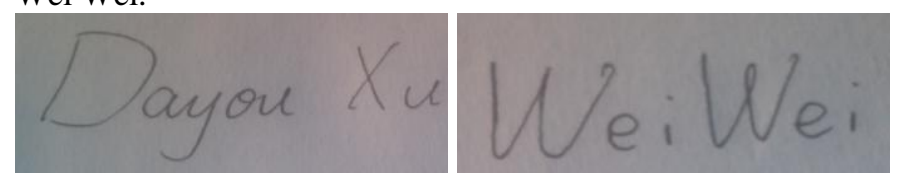

\section{ACKNOWLEDGMENT}

I would like to express my sincere heartfelt thanks to Wei Wei, for her invaluable advice and precise modification, and I admire her knowledge and her personality.

\section{REFERENCES}

[1] Bin Feng,Fenghua Zhao,Shuhua Wang. "Application of Spectral Decomposition Technique in Fluvial Sand Body Prediction," Advances in Earth Sciences.J.China,vol.5 ,pp23-25,May 2012.

[2] Chunfeng Yang,Hong Zhang,Xiaomin Tian. "Coherent data processing techniques and application in fine structure interpretation," Geophysical Prospecting for Petroleum.J.China,vol.S1,pp55-58,2004.

[3] Wei Yang,Zhenhua He,Xuehua Chen. "Application of threedimensional volumetric curvature attributes to fault identification,"Progress in Geophysics.J.China,vol.1,pp110115,2006

[4] Lei Li,Yongmin Wang,Junwei Wang. "Application of coherent data volume on fault interpretation at Qingbei area,"Petroleum Geology and Engineering.J.China,vol.4,pp36-38,2008.

[5] Jian Wu,Fanhua Li. "Prediction of oil-bearing single sandbody by 3D geological modeling combined with seismic inversion," petroleum exploration and development .J. China,vol.5,pp627627,2009 .

[6] Qiyang Zhou,Zhenhua Yan,Yangdong Xu. "Structure Modeling Using PETREL Software,"Journal of Yangtze University(Natural Science Edition).J.China,vol.2,pp62-64,2011.

[7] Songming Wang. "Application of well-seismic technology in the fault interpretation,"Journal of Oil and Gas Technology.J.China,vol.9,pp72-76,2011. 